\title{
Participatory Action Research: Reflections on Critical Incidents in a PAR Project
}

\author{
Betsy Santelli \\ Beach Center on Families and Disability, University of Kansas \\ George H. S. Singer \\ Graduate School of Education, University of California at Santa Barbara \\ Nancy DiVenere \\ Parent to Parent of Vermont \\ Connie Ginsberg \\ Family Connection of South Carolina \\ Laurie E. Powers \\ Oregon Health Sciences University
}

\begin{abstract}
This article describes a participatory action research (PAR) project designed to evaluate Parent to Parent programs in five states in the United States. The PAR team consisted of two groups: 1) parent leaders of programs that create one-to-one matches between help-seeking parents of children with disabilities and volunteer supporting parents and 2) university-based researchers. Based on a narrative record of the project, critical incidents are presented along with reflections of factors that contributed to the success of the project overall. The project successfully gathered evaluative data about Parent to Parent self-help programs. Results of both the PAR project and the study it created are presented. A process of developing a shared understanding of the programs and of the purposes for evaluating them, along with an ongoing willingness of parents and researchers to compromise, led to creative solutions to
\end{abstract}

We wish to acknowledge the other members of the Parent to Parent Consortium Team: Judy Ainbinder and Judy Colla, Hood Center at Dartmouth-Hitchcock Medical Center; Lynn Blanchard Ph.D., Jan Combest, Nancy Micca, and Michael Sharp M.D., Family Support Network of North Carolina at the University of North Carolina at Chapel Hill; Patty Gerdel and Bonni Pennie of Kansas Parent to Parent; Esther P. Lerner, Janet G. Marquis Ph.D., Sara Squires, and Ann P. Turnbull Ph.D., Beach Center on Families and Disability at the University of Kansas; Bev Parry and Maryellen Sullivan, Parent to Parent of New Hampshire, and Jo Yoder, Parent to Parent of Vermont. The research reported here was funded in part by a grant from the U.S. Dept. of Education, National Institute of Dis ability Rehabilitation Research, with the Dartmouth Medical School: Grant H133630083-94. The views stated in this report do not necessarily reflect those of the funders.

Address correspondence and requests for reprints to Betsy Santelli, Beach Center on Families and Disability, University of Kansas, 3111 Haworth Hall, Lawrence, KS 66045. E-mail address: betsy@doleasi.ukans.edu. difficulties that arose in meeting the needs of researchers and parents. As a result of the project, published data from a controlled experimental study are now available showing that Parent to Parent assisted parents in developing more positive views of their circumstances and a stronger sense of efficacy in coping with family and child challenges. Interviews indicated some of the reasons why Parent to Parent succeeds and fails. The study also revealed operational problems in the programs that were previously unrecognized. As a result of the project, the participating programs have made several changes to improve their services and they have begun to use the research data to support their legitimacy to funders and to expand their services to new populations.

DESCRIPTORS: participatory action research, parents, children with disabilities, self-help, evaluation

The purpose of this essay is to present a case study of a participatory action research (PAR) project. It brought leaders of regional self-help programs for parents of children with disabilities together with a team of university-based researchers for the purpose of evaluating the efficacy of Parent to Parent programs in five states. We have adopted a framework presented by Bruyere (1993) to provide a functional definition of participatory action research. According to this view, PAR is an approach to applied social research that seeks to (a) define relevant issues for individuals with special needs and their families, (b) find solutions to the identified problems, and (c) ensure that the research-based solutions are meaningful, useful, and actually 
make a difference in the lives of families affected by the disability experience (Bruyere, 1993). With its special emphasis on useful outcomes, PAR is an approach to research that encourages researchers and beneficiaries (in this case individuals with disabilities and their families) to come together to identify the problem to be investigated and then to collaborate throughout every phase of the research, dissemination, and utilization process. The following incidents, drawn from notes for a case study and discussion, present the joint efforts of parent leaders and researchers to create a shared research project that would be relevant and make a difference to Parent to Parent organizations and the families they serve. This essay combines some of the features of a case study and some of a discussion paper. It is organized loosely around the sections of a research report. However, instead of providing the normal detailed information in a research report, we discuss the questions, problems, and team processes that arose at each stage of the project. In order to choose critical incidents, we reviewed verbatim notes of all the team meetings and telephone conferences over the 3-year life of the project. Incidents were selected based on their representativeness of common processes or because they describe pivotal moments in the project.

\section{Parent to Parent Programs}

Parent to Parent programs represent an important grass roots movement to provide forms of family support that are often not available through formal services for families of children with disabilities. Since the early 1970s, Parent to Parent programs have been providing emotional and informational support to parents by carefully matching a trained and experienced veteran parent of a child with special needs with a referred parent who has a child with similar needs and who is seeking support from another parent. Because the two parents share so many common family and disability experiences, the support offered through the one-to-one match is often especially meaningful. A recent national survey of Parent to Parent programs revealed over 500 local and statewide Parent to Parent programs in the United States, providing support to over 35,000 parents (Santelli et al., 1995). These programs serve parents of children with developmental disabilities, chronic illness, and other special needs. Although parents who participated in the national survey anecdotally indicated that the Parent to Parent support they received through their Parent to Parent programs was helpful to them, until recently there were no formal evaluative data about the impact of Parent to Parent support on the referred parent.

\section{Initial Stages and Participants}

Parent to parent leaders. Our mutual work as partners in research began in April 1992 at the 7th International Parent to Parent Conference when a small group of parents and researchers met with Ann Turnbull, Ph.D., to talk about the need for quantitative data on the effectiveness of the one-toone Parent to Parent match. Parent leaders initiated this discussion. They were directors of statewide programs in three states. They had been with their organizations from the beginning and had shepherded them from small voluntary organizations to larger statewide programs with both volunteers and a few paid staff members. This initial informal group was small, three parent leaders and two researchers. Later the group elected to expand its membership and convene an initial meeting to advance their plan. These parents believed that if data were available that validated what parents had been informally saying about the value of Parent to Parent support, these data would be useful in convincing potential funding sources and referral sources about the importance of one-to-one Parent to Parent support. They believed that evaluation research might help them to establish the legitimacy of their grass roots programs. Such data might mean that Parent to Parent support would become more widely available to families, an important goal for Parent to Parent program directors. From the very first tentative discussions, the parent leaders were initiators and leaders of the project. The expanded group was made up of parent leaders from five states in three regions of the country: the Midwest, New England, and the South East. With the help of their initial academic contact person, they invited researchers to join the group. At least two of the parent leaders were also academics and they were able to play a dual role on the team.

University-based researchers. With the guidance of the directors of the Beach Center on Families and Disabilities, a group of researchers from universities located in or near these five states convened a meeting with the parent leaders in the summer of 1992. By design, the researchers had previously conducted family focused research and most had personal experience with disability in their families or in their own lives. The researchers had published studies using a variety of methodologies so that several major methods of research that are commonly used in the special education and disability literature were available to the group. This mix of methodological expertise was important because we eventually designed a study using a 2 group experimental design with quantitative 
measures, a qualitative interview study, and a case study of our own team processes based on verbatim notes of meetings and interviews of team members. In preliminary discussions, the initial group of parent leaders suggested that one of the academics should be designated project leader (George Singer, Ph.D.) and the home site for developing a joint grant proposal to study the efficacy of Parent to Parent self-help programs should be located at the Dartmouth Hitchcock Medical Center. The membership on our team grew from five to more than a dozen, with equal representation of parents and researchers. It is also important to note that one researcher was a pediatrician who represented an important referral source. Pediatricians were a group that the leaders particularly hoped to influence with an evaluation study and thus, the physician member of the team was able to talk to the group about the kinds of data that would be most salient to his colleagues around the country. Two other members of the team had worked with foundations and state legislatures and were similarly able to discuss the kinds of information that would likely be listened to by these potential funders.

Developing a PAR team. It is fairly common that, in university-based research projects, the members of a team are all trained as professional researchers. Even if they have not worked together, they all share a common body of technical knowledge, as well as the norms and roles involved in conducting research. Similarly, in evaluation research, evaluators often will convene meetings of key stakeholders and meet regularly with the group that has contracted for an evaluation. Traditionally, however, evaluators are the chief designers and decision makers who chose their own methods. Because these traditional approaches sometimes do not involve diverse teams of academics and nonacademics, the process of team building is often not given much deliberate attention. But when the research team consists of consumers of a study, as well as academics, and they equally share decision making authority in all stages of the project, the process of team building is not so automatic. A shared vocabulary, social protocol, and roles cannot be taken for granted. It was important to spend a good deal of time developing a shared understanding of Parent to Parent programs, their mission, and values. From the beginning the researchers made it clear that they would carefully listen to the parent leaders with the aim of developing a consensus about the purposes, methods, and targeted outcomes of an evaluation project. It also, in retrospect, seems important that there were equal numbers of researchers and leaders in the group and that the researchers had had pre vious experience in collaborating with families. We also developed a shared sense of the roles that leaders and researchers would play in the process. These key steps in the process are described in more detail in the dis cussion section below.

\section{Developinga Study}

Research questions. Because our PAR team wanted to evaluate the outcomes of one-to-one Parent to Parent support, the purpose of our second meeting was to design a research study to measure these impacts. In order to define our research questions, we started by looking carefully at the goals and objectives of Parent to Parent programs and their perceived outcomes. The parents on our team led an initial discussion about the impact of Parent to Parent support on referred parents. They movingly spoke of their own and many other parents' personal experiences. The researchers carefully listened and asked questions to clarify their understanding about the perceived importance of Parent to Parent. It was important at this stage that the researchers remained in a listening and questioning mode. The listening was active and involved follow-up questions, discussions of ideas, requests for more information, and a process of ongoing clarification. The team leader served as a facilitator rather than a director.

Based on this discussion, our PAR team agreed that the goals of Parent to Parent support are as follows:

- To increase the emotional support that is available to parents who have a child with special needs.

- To increase the informational support that is available to parents who have a child with special needs.

- To provide this emotional and informational support by offering parents a one-to-one match with a veteran parent.

In order to be more specific about what we meant by emotional support, informational support, and the oneto-one match, we defined them, based on the parents' descriptions of Parent to Parent support, as follows:

- Emotional support was defined as having five different components: (a) a sense of having a reliable ally; (b) a sense of empowerment; (c) a sense of social support; (d) a sense of being able to cope; and (e) acceptance of family and disability challenges. The construct "reliable ally" is a specific and discrete form of social support in which one person believes that there is always someone to call on who will be supportive in a time of 
need, even if there is little on-going contact (Cutrona \& Russell, 1990 ). It is a subset of the larger construct of social support. We decided to try to develop a measure of this construct and to use established measures of social support. As described below, our efforts to develop this particular measure did not succeed in the time period of the grant.

- Informational support was defined as knowledge about services for the child with special needs.

- $\quad$ The one-to-one match was defined as at least four contacts from a veteran parent during an 8 -week period.

Using these definitions of the program goals, objectives, and key terms in Parent to Parent, our PAR team identified seven different evaluation questions to answer, three outcome questions and four process questions.

The outcome questions were:

1. What is the impact of the one-to-one match on referred parents' (a) sense of having a reliable ally, (b) sense of empowerment, (c) sense of social support, (d) sense of being able to cope, and (e) acceptance of the disability issues?

2. How do parents rate the value of Parent to Parent?

3. What is the impact of the one-to-one match on referred parents' progress in meeting their need that they first brought to the program when asking for assistance?

The process questions were:

1. How does the number of contacts with the supporting parent affect the referred parents' satisfaction with Parent to Parent?

2. How does the parent's income, educational level, age of the child and the number of years that the parent has known about the diagnosis affect the impact of the one-to-one match on referred parents?

3. According to consumers of Parent to Parent supports, what makes the Programs effective?

4. When Parent to Parent does not help parents, what are the reasons?

Research design. Once we specified our research questions, we turned our attention to address how we were going to carry out this study to yield data that would be respected by (a) other re searchers, (b) funding sources for Parent to Parent pro grams, and (c) those who might refer parents to Parent to
Parent programs, particularly physicians. We knew that our research design needed to be rigorous so that our findings would be convincing and useful. Determining the research design was our biggest challenge as a PAR team-and one that nearly led to a dissolution of our effort.

At first, the researchers, based on their formal training and experience in research methods, presented what they thought would be the strongest research design: An experimental group design with a waiting list control group and an intervention group and a qualitative analysis of consumers' perceptions of the programs. The parents in the experimental group would receive a Parent to Parent match. The parents in the control group would not or, alternately, would wait up to a year for the service. We would follow each group of parents for 1 year and ask them to complete some written questionnaires several different times over the course of the year. Then we would look for any differences between the groups' responses to the questionnaires, and these differences could be attributed to whether the parent had participated in a one-to-one Parent to Parent match. The qualitative study would be conducted after we had collected a year's worth of datausing quantitative means. Based on parents' responses on a questionnaire about the helpfulness of parent to parent, we would create one group of parents who found it helpful and one group that did not. The participants would also be drawn from each state represented in the study. We would interview parents about their experiences with Parent to Parent and obtain their views on why it works and why it does not.

The parents on our PAR team were very concerned about the researchers' proposal to use an experimental design with a waiting-list control group, believing that it would be unethical to deny a parent in the control group immediate Parent to Parent support for the sake of the research. They knew from first-hand experience the importance of Parent to Parent support, and they were not comfortable in denying this support to parents who had indicated their need for it.

We explored other options: Comparing parents from two different communities, one community that had a Parent to Parent program and one that did not; or solely gathering qualitative data by interviewing parents over time. Comparing parents in two different communities was ruled out because the communities themselves might be different in terms of available services and supports for families, making it hard to sort out which differences were due to Parent to Parent. We struggled to find a solution to our need for a scientifically rigorous research design that was also sensitive to the needs of 
families. Because we were asking a question about whether Parent to Parent works and, implicitly, whether it is likely to work in other programs and regions, the researchers urged the parents to adopt an experimental design and quantitative methods that produce evidence with some claims for generalizability. In part, this emphasis was due to the fact that important targeted consumers for the research would be physicians who are used to reading and making decisions based on group comparison research. Because we also wanted to learn more about the fundamental factors that make Parent to Parent succeed and fail, researchers also recommended the use of qualitative methods to capture the viewpoints of parents who are the consumers of Parent to Parent services.

One parent on our PAR team clearly demonstrated the central importance of the control group issue when she indicated that if the research design required some parents to be denied the Parent to Parent experience, then the Parent to Parent program she directed simply would not be able to participate in the study. Without a research design that was family-sensitive, our study would not have the backing of the parents and their Parent to Parent programs on our PAR team; yet without a wellrespected research design, our study would probably not get the funding we needed to carry it out, and/or our results would not be viewed as credible by many researchers and by potential funding and referral sources for Parent to Parent. The tensions centered on the conflict between what was best from the researchers' point of view and what was best for the parent leaders who wanted to remain true to the mission and values of their programs. These disagreements, although friendly in tone, had brought us to a very difficult place. At this point, it became necessary to go into a problem solving mode in which we tried to preserve the goals of both sides through careful listening and thorough exploration of alternatives. At this point, the previous interactional work we had done to build a team paid off.

Because we had (a) taken time to get to know one another, (b) shared our enthusiasm for our proposed study, (c) realized and appreciated the mutual commitments that we each had to Parent to Parent and to quality research that would be respected and make a difference, and (d) developed a full respect for the expertise and perspectives that we each as PAR team members brought to our joint effort, we were able to develop a creative three-part compromise to our dilemma.

As uncomfortable as the experimental/control group design was for all of us, we decided parents in the control group would not need to wait for a full year before being matched because parents report that they experience the impact of Parent to Parent support right away and often immediately after the first contact. The parents on our team advised the researchers that most matches realize a significant impact in the first 8 weeks of the match. The researchers, even though they recognized that a 1year time period to compare the responses of parents in the experimental and control groups would make for a stronger study, agreed to the shorter time period for parents in the comparison group to wait until they would be matched.

For the second part of our compromise, we decided that no parent who wanted to be matched right away would be denied that opportunity. Parents who did not want to risk being assigned to the control group that waited for 8 weeks before being matched would be matched right away, and they would not be a part of the study. Although we recognized that this solution might mean that parents for whom Parent to Parent support might make the most difference would not be a part of the study, ethically we believed in the greater importance of a research project that would not harm parents in any way.

A third part of our compromise solution was the commitment that, as a part of our research effort, we would work to bring Parent to Parent into communities that, without the research, would not have a Parent to Parent program. Throughout this process it was necessary for both groups to compromise and to actively explore new options. The researchers found themselves explaining and interpreting technical research concerns to the parents whereas the parents served as informers and advocates for their values and mission.

The parents talked about what typically happens in a Parent to Parent match and mentioned that most matches have at least four contacts over an 8-week period. Therefore, for the research study to require a minimum of four contacts during the first 8 weeks of the match, it would not be changing what typically happens in most matches.

We also needed to decide the number of parents to involve in the study and methods for recruiting them. The researchers on our PAR team wanted to recruit a large number of parents because the study would have more power to detect the impact of the program with larger numbers. The parents on our team indicated what was realistic in their home states. Without the advice of the parents, the researchers might have promised to include more parents than realistically possible. Without the explanation from the researchers about the importance of a large sample, the parents might have limited the size of the study and thus reduced its power to detect group differences. We decided that an ideal study would recruit 540 
parents across the four sites over the 3 years, or 135 parents per site. In the end we fell somewhat short of this goal with 200 parents recruited for a first-year pilot study and another 200 for the final evaluation study.

Measures. Once we had determined our basic design, we went on to outline some of the remaining activities. We had talked from the beginning about measuring the impact of Parent to Parent, but we now needed to agree on how to measure Parent to Parent support: From a quantitative researcher's perspective, we needed to be sure that we were measuring the same constructs for each participating parent. And yet no two Parent to Parent matches are the same. Thus, our challenge was to figure out how to impose the necessary consistency required by this kind of research on the intimate, flexible, individualized, and personalized experiences that are at the heart of Parent to Parent. Again, our shared vision about and commitment to this study allowed us to find a creative compromise.

Based on what we had learned from the parents about the perceived outcomes of Parent to Parent support, the researchers on our PAR team presented a number of instruments that we could use to measure the kinds of outcomes that the parents had described. The researchers recommended which of these instruments they believed were the most psychometrically sound and had been used in other studies about families of children with special needs. The team reviewed all of these instruments. Parents were concerned that the total package of assessments would not require a lot of time to complete and they did not want measures that would focus parents' attention primarily on negative experiences.

The researchers reviewed each instrument and tried to translate psychometric concerns into plain language to make the technical selection of instruments understandable and create the opportunity for parent input. The researchers noted that some of the most well respected instruments have been used for many years, but they don't incorporate a positive perspective on the family. We were struggling with the competing preferences for using respected and validated instruments and choosing measures that were family-friendly and wouldn't focus parents on the most negative parts of their experiences with parenting a child with a disability. This discussion brought to light a previously unspoken philosophy of the programs. It became clear that a basic premise of the parent leaders, also shared by the researchers, was that parents could, and often did, come to terms with a child's disability. "Coming to terms" meant attaining a positive attitude about the child, family and future bolstered by actively accommodating to the child's needs.
The parent leaders, of course, recognized the many difficulties that parents face and had, in fact, listened to help seeking parents daily for a number of years. Yet they believed that an important value of Parent to Parent was to point parents in the direction of hope and acceptance. Matched veteran parents were meant to be people who had themselves come to terms with similar challenges. Consequently, parents wanted to select measures that would measure the positive presence of acceptance and hope. We also wrestled with how to include paper-andpencil research instruments into the otherwise intimate way that Parent to Parent matches are made.

We settled on six instruments. Three previously developed and published instruments were used: The Kansas Inventory of Parental Perceptions (KIPP) (Behr, Murphy, \& Summers, 1992), a measure of positive attitudes about a child with disability in the family; The Family Empowerment Scale (FES) (Koren, DeChillo, \& Friesen, 1992) used, to assess perceived changes in parent's sense of being empowered and the Social Provisions Scale (Cutrona \& Russell, 1990), a measure of perceived social support and its functions. We also developed three other measures. The first was the Parent Coping Efficacy Scale (PCES) (Blanchard \& Powers, 1998) a measure of parents self-efficacy in dealing with challenges posed by their family and child with a disability. Over the course of the research project, psychometric analyses and correlational studies supported the PCES as a reliable instrument with initial evidence for convergent validity. We also tried to develop a second measure of reliable alliance but did not succeed in creating a psychometrically sound instrument during the life of the project. The third questionnaire asked parents about the helpfulness of Parent to Parent, to what it extent it helped attain what they wanted when they first contacted the program, and information about the number of contacts and their form. We hoped that the new instruments would particularly help Parent to Parent programs in their own program evaluation, and that they would be useful gifts to Parent to Parent from the researchers. At a later stage we worked together on designing a qualitative study including its design, interview protocols, and analyses. It further explored parents perceptions of why Parent to Parent did and did not work. This study (Ainbinder et. al., 1998) did reveal that some parents developed a reliable ally in their matched helping parent. As we completed our planning process, one of the parents on our PAR team commented:

It was clear from the beginning that we would come to decisions together about what and how we 
would study Parent to Parent. Through compromise. It grew clear that no one had an agenda other than trying to understand this model of support.

An example of one such compromise arose when some of the researchers were interested in using specific measures in order to add to a program of their previous studies. When, however, parents decided these instruments were too negative in tone, the academics dropped their request to use them.

\section{Preparing a Grant Proposal}

Once we had made the difficult decisions about the design of our study, we then spent the next two months collaboratively working by telephone, fax, and e-mail to prepare the grant application that we hoped would fund our study. Roles during this grant-writing period were determined by personal expertise and preferences. Researchers and parents worked together to prepare all aspects of the proposal.

As a part of our proposal, we needed to develop a budget for the whole project, as well as for each of the five Parent to Parent sites. Because our partnership was new and the researchers were accustomed to funds coming to universities, the first draft of a budget included only funds for the three universities and no funds for the Parent to Parent programs. And yet the Parent to Parent programs had played and would continue to play a significant role in the study and would be taking on new activities. Because the parents on our team felt comfortable with our entire group and had faith in the intentions of the group, one of the parents spoke up about the budget. And just as soon as she pointed out the omission, the researchers each committed themselves to finding a way to reallocate the funds so that a subcontract could be awarded to each Parent to Parent program. Including Parent to Parent programs in the budget, on paper, and in actuality, sent a strong message of true partnership and validated the contributions that Parent to Parent programs had already made and would continue to make to the project. We also discovered that because we had used PAR as a process for planning the study and preparing the proposal, obtaining letters of support from key parent leaders was much easier. Parent leaders not on the PAR team had some of the same uneasy feelings about the design of the study as those with which we as a team had wrestled; however, because parents were a part of the team representing the interests of parents, parent leaders outside the team were willing to write support letters. We submitted our proposal in October of 1992 and funding began in July 1993.
Conducting the study. We met together again as a group in July 1993 to hammer out the details of the study and our procedures for working together across sites. We collaborated over 2 days to (a) map out specific procedures for recruitment, (b) develop protocols and letters for enrolling and communicating with parents, and (c) design a system that would guide our collection of data.

We talked at length about a variety of ways to recruit parents to participate in the study. Finally we decided to recruit parents through existing Parent to Parent channels, and the parent team members were optimistic that these channels would easily yield enough parents. It is important to note that by this time an atmosphere of partnership marked by humor and friendliness came to characterize the group. For example, the researchers, who had more experience in recruiting parents for research studies, and knew how difficult it sometimes could be to find parents who were willing to participate, were not so sure it would be possible to recruit the ideal number of parents for the study. Some of the researchers bet some of the parents a gallon of their region's famous ice cream that recruiting would take longer than the parents predicted. In the first year, the researchers were overjoyed to lose the bet. The parents won, perhaps because Parent to Parent program directors recruit parents all the time, and they know which recruitment strategies work. This incident demonstrates the informal culture that developed in the team.

We also made many decisions on what to say to parents in our telephone conversations and in our letters. The parents on our team urged us to develop telephone and written protocols that were not too formal and filled with the language of researchers. The researchers pointed out, however, that we did not want protocols that were so family-friendly that the interactions the parents had with the researchers became a support that could contaminate the data. Based on many open and candid discussions about what was ideal and what would actually work, we jointly prepared all of the questionnaire packets, informed-consent forms, cover letters, and promotional materials. These materials had quite a different look than those typically prepared by researchers alone, and this new look may have made it easier for parents to agree to be in the study. We also worked to develop a system of recruitment and enrollment that would not disturb what the local Parent to Parent programs were already doing with their own recruitment efforts outside of the study. One researcher related:

Not only do parents help the researcher with the substance of the research but also with the style or 
tone of the research. Our telephone protocol, our cover letters, and, indeed some of our instruments themselves have a much friendlier feel to them because of the input of the parents on our team.

The data collection methods that our PAR team used in the study included the five written questionnaires for all participating parents, with parents in both the experimental and the control groups completing these questionnaires at precise time intervals both before and during their one-to-one match. Questionnaires were mailed to parents before they contacted a Parent to Parent program, 2 weeks after a match was made, and then 2 months later. We also collected demographic information about the families represented in our study so we could learn more about who was participating in Parent to Parent. And we asked parents to tell us at the beginning and the end of the study about their needs, then how well their needs had been met, their stress levels, and then their satisfaction with Parent to Parent.

The qualitative study. In order to obtain qualitative data that preserved the language of consumers and expressed their personal experiences with Parent to Parent, we randomly selected 24 parents in the intervention group who completed the study. Twelve had reported on one of our measures that Parent to Parent was helpful and another 12 reported it was not helpful. An interviewer, after obtaining consent, phoned these parents and interviewed them for an average of 1 hour using an interview protocol designed to elicit their views about how Parent to Parent helped or did not help and what were the components of Parent to Parent that contributed to their overall judgments of the program.

\section{Analyzing the Data}

Once the study was underway we continued to convene the group by telephone and in two annual group meetings. Whenever a substantial amount of data was entered into the computer, we conducted preliminary data analyses. The researchers explained the meaning of the statistical analyses to the parents who became increasingly knowledgeable over time. The parents, because they had a more intimate understanding of the nuances of Parent to Parent support asked the researchers to conduct new analyses to ask about new questions that came to mind, once they better understood what could be done with the data. They became interested, for example, in running analyses that showed the relationships between program inputs and outcomes. Parents were particularly interested in why $20 \%$ of parents reported that Parent to Parent was not helpful. They later used this information to change practices in their organizations. Having the perspectives of both the researchers and the parents helped us more accurately to interpret the findings.

\section{Findings and Dissemination}

The findings from our experimental study indicate that involvement in Parent to Parent, for the subjects in our study, was associated with significant increases in positive attitudes about family circumstances and wellbeing, as measured by a subscale of the KIPP; significant gains in solving the problems that parents bring to the Parent to Parent; increases in perceived ability to cope with family and child needs among parents who initially do not feel able to cope well, as measured by the PCES; and that $80 \%$ of parents who used the service rated Parent to Parent as helpful. We did not find significant effects on a measure of empowerment nor on a measure of social support. The finding of no change in perceived social support appeared to be due to a ceiling effect; that is, the majority of parents in the study perceived themselves as having adequate levels of social support prior to contacting Parent to Parent. Follow-up analyses indicated that participating parents did show gains on one of three subscales of the FES, a scale focused on empowerment to deal with one's own family, but not on two other subscales so that the total scores used in our analyses did not detect significant change on this variable. These finding are reported in detail in a journal article (Singer, et al., in press).

Some of our process measures, both quantitative and qualitative, proved to be very useful to the five participating Parent to Parent programs. Over time we began to realize that Parent leaders' original perceptions of the efficacy and outcomes of their services were based primarily on the accounts of parents who were significantly affected by the program. This group of parents had relatively high levels of involvement with their matched parents and talked about the experience as one of major importance in their lives. However, our data showed that these parents represented, at most, $20 \%$ of consumers. The majority of consumers used Parent to Parent by making brief contacts, with $60 \%$ only making one or two telephone contacts with their matched parent in the first two months and many who never contacted a match after talking to the Parent to Parent coordinator. We also learned that many matches took much longer than the leaders had thought so that sometimes helping parents did not contact help-seeking parents for as much as three weeks from the initial call to the program. Our interview study revealed that one major reason that some parents did not find Parent to Parent helpful, $20 \%$ of our sample, was that they did not feel that they were compatible with their matched parent. 
Other logistical problems included failure of experienced parents to call back the help seeking parents and problems of finding mutually acceptable times to talk. These findings lead the parent leaders on our team to introduce new practices into their programs as described below.

The qualitative study also provided insights into what makes Parent to Parent work for the $80 \%$ who did find it to be helpful (Ainbinder et al., 1998). A thematic analysis of the interview material suggested that the program was effective in reducing parents sense of is olation in regard to their child. Most already had social networks that provided other kinds of social support but not the kind provided by Parent to Parent, an individual, and personal contact between two parents in similar circumstances. It gave them the experience of talking to someone who had been through similar experiences and who thereby seemed to especially understand their concerns. The authority that lived experience lent to the helping parents allowed the help seekers to gather believable information about problems of every day life with a child with special needs as well as information about services. Even brief contacts gave parents the sense that there was one individual, the matched parent, or one service, Parent to Parent, that was reliably available to them and reliably supportive, even if they did not make frequent use of either the matched parent or the program. The interviews also suggested that a valuable aspect of Parent to Parent matches was that parents could use the telephone to make contact rather than needing to attend meetings which their busy schedules often did not allow.

Secondary analyses of the quantitative data also provided useful information. One particular finding of importance was that Parent to Parent was equally helpful to low-income and working-class parents as it was for middle-class participants. We had deliberately recruited a significant proportion of low-income and ethnic-minority parents in order to ask this question. Prior to this research Parent to Parent had primarily been identified as a movement of white middle-class mothers (Santelli et al., 1995).

Another analysis of the data from this study has provided a model of the factors that contribute to changes in parental acceptance of heir situation (Orosz, 1997). This structural equation model suggests that cognitive adaptation to a child's disability is mediated by perceived social support and self-efficacy. It suggests that the way to assist a parent to see their situation in a more positive light is to help them feel supported with the kind of social support they need, and to assist them to become more capable of coping with their circumstances.

When combined, these data suggest that several of the purposes of Parent to Parent programs are realized in practice and that they provide valued forms of assistance to parents who request help. They also offer a unique form of assistance that is not available from the formal service system in the states where these programs operated.

Another product of this study is the Parent Coping Effectiveness Scale (Blanchard et al., 1998) It is a short measure of parents' sense of their ability to effectively deal with challenges posed by their family circumstances and their child's disability.

In addition to traditional products such as journal articles and conference presentations that fulfill researchers' needs for publication and professional contribution, the team has produced more functional materials to make available to Parent to Parent programs around the country. Our more practical products include a manual on program evaluation specifically for Parent to Parent programs, a packet of materials for parents and researchers to use to present our findings and discuss the implications of our results for Parent to Parent programs nationwide, and a summary of our results formatted for easy use by Parent to Parent programs in their public awareness efforts.

\section{Outcomes}

One major purpose of PAR is to produce information that will help people with disabilities and their families to better address a problem that is important to them. Fairly immediate application of findings and practical changes are emphasized by proponents of PAR (Turnbull, Friesen, \& Ramirez, 1995). Our study has had an immediate impact on the conduct of Parent to Parent programs that serve over 2,000 parents in five states. These programs have continued to expand into new areas of their service catchment areas and have begun to more actively recruit from lower income and ethnic minority groups. From the analyses of our data, our team has learned a lot not only about the efficacy of the one-to-one Parent to Parent match, but also about Parent to Parent programmatic process issues. The research process has provided new information to parent leaders about the operation and outcomes of their efforts. One of the Parent to Parent program directors on our consortium team, described this learning experience:

One of the neat aspects of this parent-research team effort is that I always come away from our meetings feeling as though I have attended "Parent to Parent school." The fact that researchers need to know precisely what is being measured is helping us to be more thorough in how we do Parent to Parent. We helped to define for the research 
what typically happens in a Parent to Parent match, and now the research is helping us to maintain and improve the quality of the support that we at Parent to Parent want to provide to parents through the match.

Already these program coordinators have initiated new procedures to improve outcomes. They have established monitoring systems that allow them to learn quickly about matches that are, not working or matches that do not lead to more than one phone contact. They now actively aim to increase contact levels between help seeking parents and their matches so at least four contacts occur during the first 8 weeks of the match. One of our findings was that help seeking parents, after a first contact from a supporting parent, did not know who should initiate subsequent calls and so sometimes the contact with a matched parent consisted of only one telephone conversation. As a result of our findings, the participating programs have also adopted some changes in training in order to prepare helping parents to initiate follow-up contacts and to let the program know when a different match is indicated. New questions for further research have been identified and our PAR team has submitted a second grant proposal to answer these questions. Thus, a short term outcome of the study has been to improve practices in programs that serve over 2,000 families in three regions of the US.

As for assisting Parent to Parent programs to attain legitimacy and thereby receive the support of public and private funders, we are still in early stages of the process of making requests based on our research evidence. The major findings are in articles that have been published very recently. We have had requests for pre-publication drafts by leaders of Parent to Parent Programs in other parts of the US who wish to use them in making appeals to state legislators and private foundations. Despite the slow process of dissemination through traditional vehicles, participating programs have incorporated our findings into successful proposals for funding and have been able to increase their referral rates from pediatricians and hospitals. One of the programs has developed a focus on a new population of parents of premature infants and has gained full cooperation from regional hospitals in making referrals. Thus at this early stage after the completion of the project it has begun to, at least modestly, achieve some of its original goals. We expect that our studies will contribute to a growing sense of legitimacy of this important movement once they have been more widely disseminated through our presentations at national conferences, mailings to programs, and journal publications.
As we have shared our results publicly, we have done so with parents and researchers presenting jointly. The parents on our PAR team are learning to talk about research methodology, and the researchers are learning to discuss the finer points of Parent to Parent program goals and activities. Because our research effort met a real need for Parent to Parent, interest in our findings has been high, particularly among other parent leaders and professionals involved in direct service for families of children with disabilities. We have made a point of presenting jointly and as equals at national and regional conferences.

\section{Discussion}

Based on an analysis of verbatim case notes, a thematic analysis of the interview data and discussions between the members of the team, we have identified several factors that we believe to be keys to our successful PAR project. We hope these ideas will be helpful to other collaborative teams.

Key 1: A shared vision does much to reduce the differing perspectives that sometimes exist between families and researchers. Chesler (1991), Hall (1984), Turnbull and Turnbull (1996), and WhitneyThomas (1997) all speak to the importance of mergers between researchers and constituencies and establishing shared commitment and trust before these groups even specify the research questions. Turnbull \& Turnbull (1996) point out that the shared ownership and the attention to the developing partnership that come from early involvement of all parties heighten commitment, intensify collaboration, and eventually bring about more immediate and meaningful use of the research results.

Key 2: There is safety in numbers and clearly defined roles help a lot. The fact that our team had equal numbers of parents and researchers added to the comfort that we all felt with each other. Menz (1995), Morningstar (1994), and Whitney-Thomas (1997) stress acknowledging the diverse perspectives and skills that each PAR team member brings to the process and recommend that PAR teams identify preferred roles and responsibilities for each PAR team member. Researchers are dedicated to scientific rigor and are trained in technical areas. Parents' direct life experiences offer a different perspective to the PAR team effort (Fenton, Batavia, \& Roody, 1993). When these areas of expertise are clearly defined, the PAR team as a whole may make better use of the diverse skills represented on the team.

Whyte and Doe (1995) stress the importance 
of achieving a balance in PAR team membership, both in numbers and in perspective and expertise brought to the PAR effort. Everyone on our PAR team brought their own unique skills to the team effort, and roles and responsibilities were established based on these skills and preferences. The parents on the team had first-hand knowledge of Parent to Parent, both as program directors and as parents themselves of children/adults with special needs. The researchers brought many years of professional training and experience in conducting research. Although we recognized that our roles and responsibilities would vary somewhat depending on the tasks at hand, the researchers took on the following roles and responsibilities:

- Providing information about research design and research methods - what makes for solid research.

- Suggesting/leading the development of instruments that might be used in the study.

- Managing the implementation of the administration of measures.

- Running all of the statistical analyses and summarizing the data.

- Publishing the findings in professional journals.

The parent leaders took on the following roles and responsibilities:

- Providing information about Parent to Parent - how it works and what it does for parents.

- Suggesting modifications to the research design and research methods so that the study was more comfortable for parents.

- Recruiting the subjects.

- Writing about the findings in such a way as to be clearly understood by parents.

Key 3: Trust the legitimacy of consumers' input into formal research. Some researchers have expressed concerns about the validity, reliability, and objectivity of PAR efforts (e.g., Bailey, 1997). They fear that nonresearchers do not have the relevant training to decide about research design; thus research design issues may be decided more on sensitivity-to-constituency issues than on scientific rigor. They are also concerned that the close relationship between the researcher and stakeholder may damage the objectivity of a study. But our experience was consistent with that expressed by Whitney-Thomas (1997) who wrote, "issues of validity and reliability are addressed in any research and have more to do with how one carries out methodology than who is involved in deciding what methods are used" (p.191). Bruyere (1993) and Whyte and Doe (1995) point out that an effective PAR team will address questions of scientific rigor just as would any team of researchers, with the ideals of traditional standards of research being balanced with the enhanced sensitivity and relevance of the project outcome. Reason (1994) adds:

"A key notion here is dialogue, because it is through dialogue that the subject-object relationship gives way to a subject-subject one, in which academic knowledge of formally educated people works in a dialectic tension with the popular knowledge of the people to produce a more profound understanding of the situation" (p. 328).

Key 4: Compensate all team members and recognize, correct and learn from the "Oops Factor. " Although researchers and families who have participated on a PAR team value and feel compensated in an abstract way for the experience itself, there is no question that effective implementation of PAR demands time, energy, and fiscal resources (Fenton, et. al., 1993; Menz, 1995; Turnbull, Friesen, \& Ramirez, 1995). WhitneyThomas (1997) underscores the importance of equal compensation for equal contributions and suggests that PAR teams revisit compensation issues throughout the PAR effort. In our case we made an initial mistake in early planning in not budgeting for costs to the Parent to Parent programs. Fortunately we caught it early and corrected this problem.

Key 5: Encourage all PAR team members to take on the role of expert by sharing their own expertise, perspectives, and skills. When there is an easy sharing of expertise, perspectives, and skills, PAR teams benefit from the collective knowledge of the group, and the group finds greater meaning in the research results. A parent leader explained:

For me the most powerful aspect of this entire process was how valued our parent perspective was.... When I think of the work we did on the conference calls, and how productive it was because we all demonstrated respect for each other and each other's opinions... Questions raised were answered with explanations that provided a context to understand a different perspective.... We molded and crafted this study together.

A researcher added:

One of the biggest advantages of having parents on the parent-researcher team is that we as researchers get a perspective that we don't normally have. We have a chance to learn from the parents about what they think is helpful about 
Parent to Parent. This information helps us to make better recommendations to our team about the methodology of the study and the instruments to use so that we end up with a study that is meaningful and relevant to the parents.

\section{Challenges of PAR}

As our first experience as a PAR team came to a close, we reflected on some of the challenges of PAR in our mutual effort to determine the efficacy of the one-to-one Parent to Parent match. The advantages have been described above in terms of a mutually rewarding and ultimately effective team effort. The challenges include some of the following:

- PAR efforts, because they are democratic, take more time than projects completed in isolation. Group decisions can only be made after the team has had time to process and learn from the diverse perspectives of the PAR team. When planning a PAR project, build in sufficient time for the group process that is important for group consensus. Funding agencies seeking to encourage PAR projects will need to allow more time for proposal preparation as well as project implementation.

- With the added time that is needed for PAR, additional funding is also necessary to support the extra planning meetings and conference calls that are crucial to the ongoing communication. Budgets will need to include funding to support PAR planning activities as well as the actual research activities.

- PAR team members also need to have a willingness to share not only in the work of the project, but also in the rewards. Presentations and papers with multiple authors often are the results of a PAR effort and PAR team members need to be comfortable with this reality. Universities need to support and reward the efforts and products of faculty members who are involved in PAR research.

- $\quad$ Products of PAR efforts need to be available in a variety of formats. Journal articles and conference presentations are not accessible to most families.

- In order for PAR to be replicated more widely, there is a need for comprehensive documentation and validation of PAR efforts and best practices.

Our project and the study it produced had some limitations. Our team membership was limited to three regions of the United States. Our sample did not include the full range of diversity found in the contemporary United States, although we did include low-income and minority parents. Another limitation is that our team only included one representative of the "system" that we hoped to influence: pediatricians, neonatalogists, hospital administrators, state legislators, and foundation decision makers. One member was a pediatrician and, although other team members had worked extensively with medical providers and state legislators, we had no team members who were presently in the roles of elected officials or foundation decision makers. It is likely that the project would have been strengthened with these members of the system included and that we might have achieved quicker results in regard to funding.

The process of compromise that characterized our planning, while resulting in creative and mutually agreed on solutions, also required both sides to give up important aspects of their work. The Parent to Parent leaders had to leave out parents in emergencies from the study sample even though this group of parents is one important constituency. Similarly, the researchers had to drop assessment instruments with long established evidence for various kinds of validity in favor of newer measures. In conclusion, we agree with Hall (1984) who wrote in his discussion of PAR in the International Review of Education, "PAR is an approach which deserves closer scrutiny by more practitioners, more support by agencies and funders, more respect for its intentions, and greater promotion of the advances made" (p. 299). Our hope as a PAR team is that our experiences and the lessons we learned along the way, will encourage others to take Hall's recommendation to heart.

\section{References}

Ainbinder, J., Blanchard, L., Singer, G. H. S., Sullivan, M., Powers, L. K. Marquis, J. \& Santelli, B. (1998). How parents help one another: A qualitative study of Parent to Parent self-help. Journal of Pediatric Psychology 23, 99-109.

Bailey, D. (1997). A panel discussion of participatory action research. Conference presentation. Division for Early Childhood. New Orleans, LA.

Behr, S. K,, Murphy, D.' L. (1993). Research progress and promise: The role of perceptions in cognitive adaptation to disability. In Tumbull, A. P., Patterson, J. M., Behr, S. K., Murphy, D. L., Marquis, J. G., Bluebanning, M. J. (Eds.) Positive adaptation to disability (pp. 151-164). Baltimore: Paul H. Brookes. 
Blanchard, L., Powers, L., Marquis, J., Singer, G. H. S., Santelli, B., \& Ginsberg,. C. (1998). The Coping Efficacy Inventory: Measuring parents perceptions of coping with a child with a disability and family problems. Unpublished manuscript. University of North Carolina Medical School, Chapel Hill, NC.

Bruyere, S. (1993). PAR: Overview and implications for family members of persons with disabilities. Journal of Vocational Rehabilitation, 3(2), 6268.

Chesler, M. A. (1991). Participatory action research with self-help groups: An alternative paradigm for inquiry and action. American Journal of Community Psychology, 19(5), 757-768.

Cutrona, C. E. \& Russell, D. W. (1990). Type of social support and specific stress: Toward a theory of optimal matching. In Sarason, B. R., Sarason, I. G., \& Pierce, G. R. (Eds.) Social support: An interactional view (pp. 319-366). New York John Wiley \& Sons.

Fenton, J., Batavia, A., \& Roody, D. (1993). Proposed policy statement for NIDRR on constituency-oriented research and dissemination (CORD). Washington, DC: National Institute on Disability and Rehabilitation Research.

Hall, B. (1984). Research commitment and action: The role of participatory research. International Review of Education, 30,289-299.

Koren, P. E., DeChillo, N., Friesen, B. (1992). Measuring empowerment in families whose children have emotional dis abilities: A brief questionnaire. Rehabilitation Psychology 37, 305-321.

Menz, F. E. (1995). Constituents make the difference: Improving the value of rehabilitation research. Menomonie, University of Wisconsin-Stout; Research, Rehabilitation and Training Center on Improving Communitybased Rehabilitation Programs.

Morningstar, M. E. (1994). PAR: Whose research is it anyway? Paper presented at the Syracuse University Consortium for Collaborative Research on Social Relations, Syracuse, NY.

Orsosz, C. (1997). Modeling positive parental adaptation to a child's disability. Doctoral dissertation, University of South Carolina. Dissertation Abstracts International, 5711 B.

Reason, P. (1994). Participation in human inquiry. Thousand Oaks, CA: Sage.

Santelli, B., Turnbull, A. P., Marquis, J. G., \& Lerner, E. P. (1995). Parent to Parent programs: A unique form of mutual support. Infants and Young Children, 8(2), 48-57.

Singer, G. H. S., Powers, L. K., Marquis, J., Blanchard, L., DiVinere, N., Santelli, B., Sharp, M.
\& the Consortium to Evaluate Parent to Parent (in press). A multi-site evaluation of Parent to Parent self-help programs: quantitative and qualitative findings. Journal of Early Intermediation.

Turnbull, A. P., Friesen, B. J., \& Ramirez, C. (1995). Participatory action research: Forging collaborative partnerships with families in the study of disability. Paper presented at National Institute on Disability and Rehabilitation Research conference on participatory action research. Washington, DC.

Turnbull, A. P., \& Turnbull, H. R. (1996). Participatory action research. In National Council on Disability (Ed.) Improving the implementation of the Individuals with Disabilities Act: Making schools workfor all of American children (pp. 685-710). Washington, DC: National Council on Disability.

Whitney-Thomas, J. (1997). Participatory action research as an approach to enhancing quality of life for individuals with disabilities. In Schalock R. (Ed.) Quality of Life: Applications to persons with disabilities (Vol. II, pp. 181-198). Washington, DC: American Association on Mental Retardation.

Whyte, J., \& Doe, T. (1995). Participatory action research: Forging collaborative partnerships. Paper presented at National Institute on Disability and Rehabilitation Research conference on participatory action research. Washington, DC.

Received: May 14, 1997

Final Acceptance: March 31, 1998

Guest Editor in Charge: Hyun-Sook Park

This research was supported by a grant from the National Institute on Disability and Rehabilitation Research to the Beach Center on Disability, Grant \#H133B30070. 